\title{
Design of Simulation Builder Software to Support the Enterprise Modeling and Simulation Task of the AMTEX Program
}

\author{
M. Nolan \\ A. Lamont \\ L. Chang
}

December 12, 1995

This is an informal report intended primarily for internal or limited external distribution. The opinions and conclusions stated are those of the author and may or may not be those of the Laboratory.

Work performed under the auspices of the U.S. Department of Energy by the Lawrence Livermore National Laboratory under Contract W-7405-Eng-48. 


\section{DISCLAIMER}

This document was prepared as an account of work sponsored by an agency of the United States Government. Neither the United States Government nor the University of California nor any of their employees, makes any warranty, express or implied, or assumes any legal liability or responsibility for the accuracy, completeness, or usefulness of any information, apparatus, product, or process disclosed, or represents that its use would not infringe privately owned rights. Reference herein to any specific commercial product, process, or service by trade name, trademark, manufacturer, or otherwise, does not necessarily constitute or imply its endorsement, recommendation, or favoring by the United States Government or the University of California. The views and opinions of authors expressed herein do not necessarily state or reflect those of the United States Government or the University of California, and shall not be used for advertising or product endorsement purposes.

This report has been reproduced directly from the best available copy.

Available to DOE and DOE contractors from the Office of Scientific and Technical Information P.O. Box 62, Oak Ridge, TN 37831

Prices available from (615) 576-8401, FTS 626-8401

Available to the public from the National Technical Information Service

U.S. Department of Commerce 5285 Port Royal Rd. Springfield, VA 22161 
Demand Activated Manufacturing Architecture Project

EMS-95136

\title{
Design of Simulation Builder Software to Support the Enterprise Modeling and Simulation Task of the AMTEX Program
}

\author{
Revision 1.0
}

Revised: December 12, 1995

Mathew Nolan, Alan Lamont, and Lychin Chang

\author{
Lawrence Livermore National Laboratory
}


TABLE OF FIGURES ................................................................................. IV

LIST OF ACRONYMS AND DEFINITIONS ................................................... V

I. OBJECTIVE

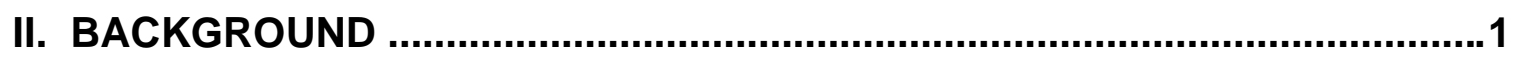

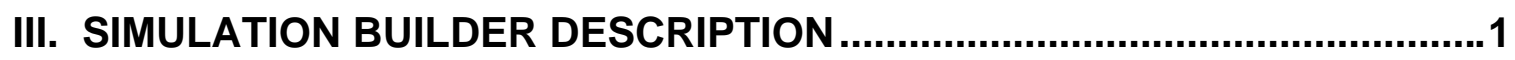

A. Module Definition ................................................................................................................. 2

B. Services and Communication Between Modules.................................................... 3

C. Method Classifications .................................................................................................... 4

D. Structure of the Simulation Builder...........................................................................5

E. Validity Checking through Module Registry ....................................................... 15

F. Model Instantiation ........................................................................................16

IV. STEPS FOR THE MODULE DESIGNER …............................................... 17

A. Creating and Implementing a New Service............................................................. 17

B. Designing a New Module .............................................................................................. 19 


\section{Table of Figures}

Figure 1: Example simulation model showing modules and their

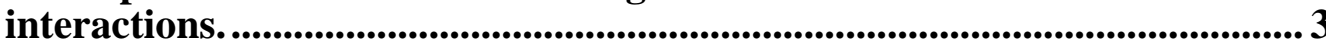

Figure 2: State of modules and registry after the modules have been constructed but before connecting

Figure 3: State of modules and registry after connecting................................................. 8

Figure 4: Example inheritance structure for module type module_warehouse.

Figure 5: Connection between modules.................................................................................. 11

Figure 6: Inheritance diagram showing the relationship between module, standard service message class, and ports............................................................. 13

Figure 7: Pointer returned from find() message is cast to the appropriate SSMC class 


\section{List of acronyms and definitions}

\section{Acronyms}

DAMA

EMS

SSM

SSMC

CAC

$\mathrm{OO}$
Demand Activated Manufacturing Architecture

Enterprise Modeling and Simulation

Standard Service Message

Standard Service Message Class

Class Attribute Class

Object-Oriented

\section{Definitions}

Conceptual terms:

\begin{tabular}{|c|c|}
\hline & Definition \\
\hline object & $\begin{array}{l}\text { A concept, abstraction or thing with crisp boundaries and } \\
\text { meaning for the problem at hand. }\end{array}$ \\
\hline object class & $\begin{array}{l}\text { Description of a group of similar objects with similar properties, } \\
\text { common behavior, common relationships to other objects, and } \\
\text { common semantics. }\end{array}$ \\
\hline object instance & $\begin{array}{l}\text { Exactly one thing of an object class. Often abbreviated to } \\
\text { object. }\end{array}$ \\
\hline attribute & A data value held by the objects in an object class. \\
\hline operation & $\begin{array}{l}\text { A function or transformation that may be applied to or by } \\
\text { objects in an object class. }\end{array}$ \\
\hline class attribute & An attribute shared by all instances of an object class. \\
\hline class operation & An operation on the class itself. \\
\hline method & The implementation of an operation in an object class. \\
\hline
\end{tabular}




\section{C++ terms:}

\begin{tabular}{|c|c|}
\hline & Definition \\
\hline class & $\begin{array}{l}\text { In } \mathrm{C}++ \text {, the keyword indicating the declaration of a user- } \\
\text { defined type. Use a class to implement an object class. }\end{array}$ \\
\hline $\begin{array}{l}\text { member } \\
\text { function }\end{array}$ & A function bound to a class. \\
\hline method & The implementation of an operation in an object class. \\
\hline instance & $\begin{array}{l}\text { What we refer to when speaking of a particular one of things } \\
\text { of the same type. }\end{array}$ \\
\hline constructor & The class method responsible for creating new instances. \\
\hline instantiate & To create an instance of some type. \\
\hline abstract class & $\begin{array}{l}\text { An abstract class defines an interface to an object without } \\
\text { exposing any implementation details. }\end{array}$ \\
\hline
\end{tabular}


SimBuilder terms:

\begin{tabular}{|c|c|}
\hline & Definition \\
\hline model & $\begin{array}{l}\text { A description or abstraction of a system developed to solve } \\
\text { specific problems or answer questions. }\end{array}$ \\
\hline simulation & $\begin{array}{l}\text { A mathematical representation of a system implemented and } \\
\text { executed on a computer to evaluate the performance of the } \\
\text { system. }\end{array}$ \\
\hline service & An atomic unit of abstract functionality an object provides. \\
\hline module & $\begin{array}{l}\text { The SimBuilder's basic unit of namable abstract functionality } \\
\text { with which modelers think about simulation models. Modules } \\
\text { make one or more services available to other modules. } \\
\text { Modules may require services of other module. }\end{array}$ \\
\hline $\begin{array}{l}\text { standardized } \\
\text { service message } \\
\text { (SSM) }\end{array}$ & $\begin{array}{l}\text { Having conceptualized a service, a modeler writes a } \\
\text { specification that specifies the given name for the service and } \\
\text { a set of input types and output types associated with it. For a } \\
\text { given service, the method that has been given a name, and } \\
\text { argument list. This specification becomes the formalized } \\
\text { standard interface definition for the service. }\end{array}$ \\
\hline service method & $\begin{array}{l}\text { A method differentiated from other methods in that we derive } \\
\text { modules classes from classes containing said methods. A } \\
\text { method of a module class that another module may wish to } \\
\text { invoke. We also refer to this as an SSM. }\end{array}$ \\
\hline $\begin{array}{l}\text { standard service } \\
\text { message class } \\
\text { (SSMC) }\end{array}$ & $\begin{array}{l}\text { An abstract class whose sole purpose is to define the } \\
\text { standardized interface corresponding to a service. }\end{array}$ \\
\hline link & $\begin{array}{l}\text { The name for the association between two communicating } \\
\text { modules. }\end{array}$ \\
\hline module class & $\begin{array}{l}\text { A class providing an implementation for a set of SSMs. A } \\
\text { module acquires its SSM interface(s) by inheriting a set of } \\
\text { SSMCs. }\end{array}$ \\
\hline message & $\begin{array}{l}\text { In the OO paradigm, sending the message } m \text { to an object } \\
\text { instance named object_inst corresponds to calling the member } \\
\text { function } m \text { of object_inst. }\end{array}$ \\
\hline register/registry & $\begin{array}{l}\text { A class may contain within itself a class attribute that } \\
\text { contains a list of instances of said class. We refer to this list } \\
\text { of instances as the registry for the class. We say an instance } \\
\text { registers itself when it adds itself to the registry. }\end{array}$ \\
\hline input name & $\begin{array}{l}\text { A name for module instance as specified by the simulation } \\
\text { user in the simulation input. }\end{array}$ \\
\hline
\end{tabular}


Names of classes in SimBuilder:

\begin{tabular}{|c|c|}
\hline & Description \\
\hline input_mod & Simulation input information for a module. \\
\hline input_ssm & Simulation input information for an SSM. \\
\hline instance_info & $\begin{array}{l}\text { Contains attributes for generic information in regards to an } \\
\text { instance, such as pointer to the instance and a reference to } \\
\text { its input_mod. }\end{array}$ \\
\hline instance_info_mod & $\begin{array}{l}\text { Instance information for module classes. Contains an } \\
\text { attribute of type input_mod, which in turn contains a } \\
\text { reference to input name. }\end{array}$ \\
\hline instance_info_ssm & Contains instance information for instances of SSM classes. \\
\hline CAC_ssm & $\begin{array}{l}\text { Class attribute class for all types of SSMC. This maintains } \\
\text { information about the instantiated objects of the class. }\end{array}$ \\
\hline CAC_mod & Class attribute class for all types of module classes. \\
\hline provider $<$ SSMC $>$ & $\begin{array}{l}\text { To acquire a type of service, a module must contain an } \\
\text { attribute of the template class provider }<\text { SSMC }>\text {. }\end{array}$ \\
\hline port_ssm & $\begin{array}{l}\text { Contains the type independent attributes needed to } \\
\text { implement links. All provider }\langle\text { SSMC }>\text { classes inherit } \\
\text { port_ssm. }\end{array}$ \\
\hline
\end{tabular}




\section{Design of Simulation Builder in support of the Enterprise Modeling and Simulation Task}

\section{Objective}

This document describes the implementation of the Simulation Builder developed as part of the Enterprise Modeling and Simulation (EM\&S) portion of the Demand Activated Manufacturing Architecture (DAMA) project. The Simulation Builder software allows users to develop simulation models using pre-defined modules from a library. The Simulation Builder provides the machinery to allow the modules to link together and communicate information during the simulation run.

This report describes the basic capabilities and structure of the Simulation Builder to assist a user in reviewing and using the code. It also describes the basic steps to follow when developing modules to take advantage of the capabilities provided by the Simulation Builder.

The Simulation Builder software is written in $\mathrm{C}++$. The discussion in this report assumes a sound understanding of the $\mathrm{C}++$ language. Although this report describes the steps to follow when using the Simulation Builder, it is not intended to be a tutorial for a user unfamiliar with $\mathrm{C}++$.

\section{Background}

The simulation task is one of the subtasks outlined in the DAMA EM\&S Task Plan for FY95-96. (DAMA-I-2-95). The EM\&S task plan for FY95-96 discusses the three primary purposes for the industry simulation: to validate the business information models, to analyze strategic innovations identified by the EM\&S task participants, and to serve as a decision and learning tool for industry partners.

Long-term goals of the DAMA project include evolving a set of questions and providing on-going analysis. Analysis will be captured in a model representation that will be simulated for validation and evaluation of business strategies relevant to the textile valuechain. As the models constantly change and are elaborated upon, we developed a generalized framework for model construction.

To assist the work of the simulation subtask, we developed a software framework, or a generalized Simulation Builder. This will support the development and validation of a number of business simulation models. The Simulation Builder constructs and executes simulation models using specifications from the user. We describe the concept and components of the Simulation Builder in the following sections.

\section{Simulation Builder Description}

The Enterprise Modeling and Simulation task will develop simulation models of the textile industry. To make these efforts as efficient as possible one could hope to construct these models from a library of pre-defined modules that represent different components of the business system (e.g. production facilities, warehousing, order processing, etc.). To construct a model the user will assemble a set of modules into a 
network. The modules communicate during the simulation run along the links of the network passing information and instructions back and forth. Modelers can build new models from existing modules, making modifications as needed to represent new behaviors.

A complete simulation modeling system will include a number of components: a library of modules that represent different elements of the modeled business system (e.g. production facilities, warehousing, order processing, etc.), structures that generate random events or save and display results, a user interface, and components that manage the threads of execution of the processes during a simulation. The Simulation Builder focuses on the framework for building the modules themselves. It provides the mechanisms to link the modules together in a network and to allow the modules to call each other.

We designed the Simulation Builder to serve two different types of users. One, the modeler who constructs particular models from a library of existing modules and two, the module designer who develops and programs the modules in the library. The Simulation Builder has been designed to make the job of both users as easy as possible.

The modeler specifies a network of simulation module instances to construct a particular simulation model. For each module the user specifies a user given name (referred to as its input name) and values for the module's parameters. In general, a module communicates with, and needs references to other modules. This communication occurs through links to other modules. To instantiate a module, the user also specifies a set of input names, one for each link the module instances contains. The type of each link indicates what information passes between the pair of connected modules.

The Simulation Builder provides the module designer with the mechanisms needed to establish communication links between modules and to validate them. When instantiating a particular model, the user will supply each module with a name, such as "Central-warehouse". When writing a module, however, the module designer does not know the names of the external modules to which the module being written will connect. The designer only knows the abstract functionality the external modules provide. The Simulation Builder provides a fairly simple mechanism for establishing references to other modules, prior to those modules being instantiated. The module designer uses a set of predefined structures for establishing the connections. These predefined standardized classes maintain all of the data needed to maintain the connections and include the generic methods needed to establish links between modules and to pass data back and forth.

Since it is possible to make errors in specifying the links between modules (names can be misspelled, or the wrong module can be specified), the Simulation Builder includes mechanisms that check for link compatibility and validity of model structure. This ensures that two connected modules exchange information properly.

The rest of this report explains the specific mechanism used by the Simulation Builder and outlines the steps that a module designer needs to follow to develop new modules within the Simulation Builder.

\section{A. Module Definition}

Modules serve as the component building blocks of a simulation model. From the software engineering standpoint, we represent modules as objects in the software. We define a module as an object that provides a set of services (implemented as methods) to 
other modules. A module may represent a set of business functions or production processes. Generally, a module represents a permanent component of the system that can receive information and instructions, act upon them, and pass along information and instructions. Thus, we distinguish a module from other objects in that a module object does not move from one place to another. For example, a warehouse object or production object can be a module since these objects "act" upon other objects and can perform certain functions. A purchase order, on the other hand, is merely a collection of information, and is not represented as a module. A purchase order is created, is transferred from place to place, may be transformed, and is destroyed. We refer the reader to EMS document \#95056, "TECHNICAL NOTE: Preliminary Requirements for Module Definitions for Simulation Builder", for a more complete description of module design.

Modelers construct a simulation model by selecting from a library of pre-defined module classes and connecting them together into a network structure. Figure 1 below illustrates a simple network consisting of four modules.

\section{Figure 1: Example simulation model showing modules and their interactions.}

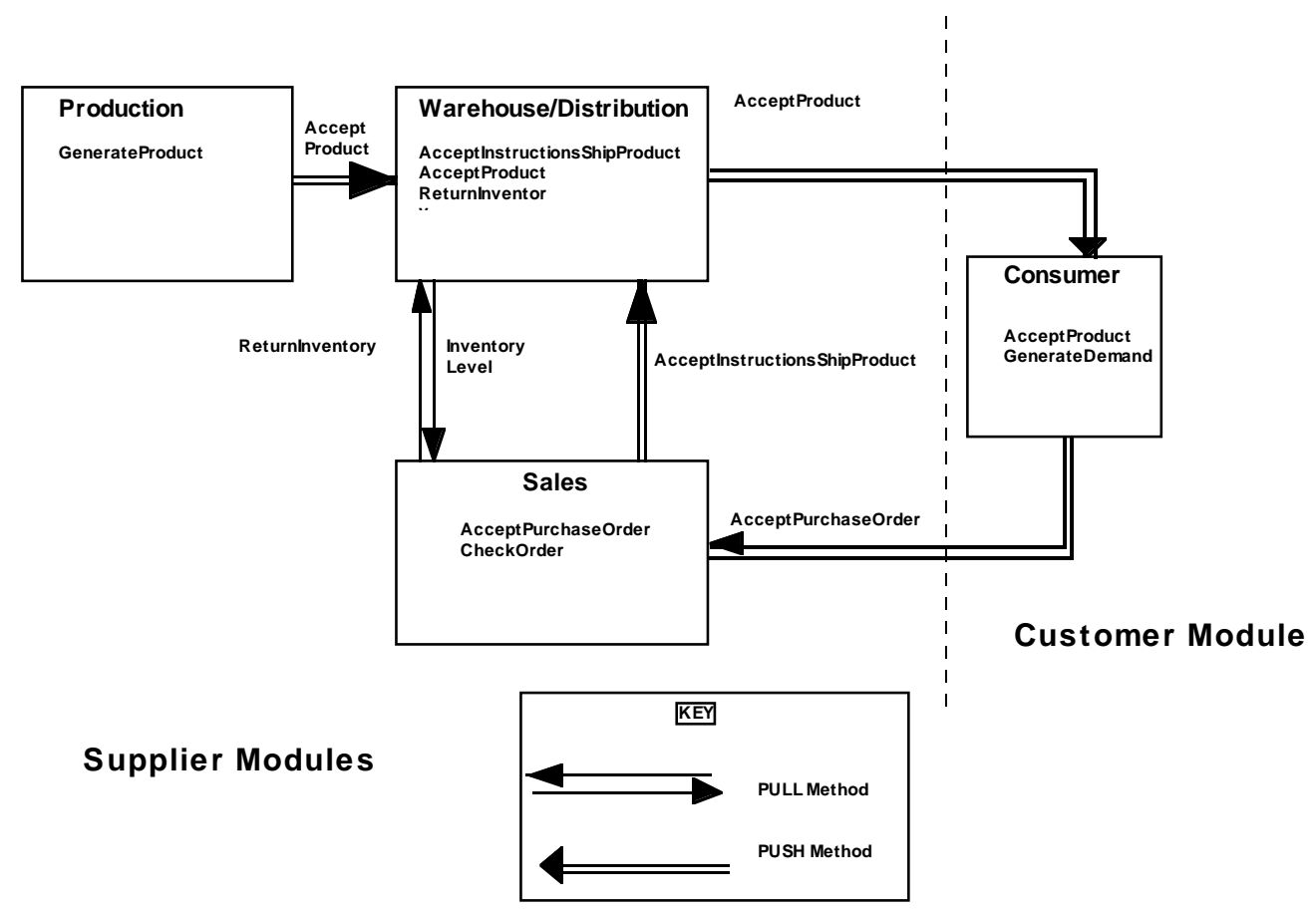

\section{B. Services and Communication Between Modules}

Communication between modules occurs when one module invokes a special method of another module. A module communicates with an external module by invoking a method of the external module. Calls obtain information from a module or send it information or instructions. The collection of methods within a module represents the services provided by the module. A service is defined as an action that a module may perform for other modules, such as returning inventory levels, or the shipping of a product. The example simulation model in Figure 1 shows the module of type Warehouse/Distribution providing the services AcceptInstructionsShipProduct(), AcceptProduct(), and ReturnInventory(). The module of type Consumer provides the services AcceptProduct () and GenerateDemand(), and the module of type Sales provides the service AcceptPurchaseOrder(). 
Following the example through, a Consumer instance GeneratesDemand() and instructs the Sales instance to AcceptPurchaseOrder(). This call causes the Sales instance to query the Warehouse instance to ReturnInventory(). The ReturnInventory() method returns the inventory level. Then, the Sales instances performs a CheckOrder() to compare the inventory level against the quantity required in the purchase order. If the order can be filled, the Sales instance tells the Warehouse instance to AcceptInstructionsShipProduct(), and the Warehouse instance will ship product. Production periodically replenishes the warehouse. In this illustration, it does so periodically without requiring instruction.

\section{Method Classifications}

The example simulation model shown in Figure 1 illustrates three basic types of method calls. For the sake of clarity, we define naming conventions for each type of method. Standard method names distinguish whether methods return information, or whether information passes to another object without a requiring a response.

The first type of method call, a PULL mechanism, represents a request by a module to another module to return data or to return a pointer to an object. The calling module always expects an immediate return from the call. An immediate return implies no simulation time elapses. A standard method name proposed for a PULL mechanism is "ReturnSomething", such as "ReturnInventory". The prefix "Return" suggests the method call expects a return value.

The second type of method call, a PUSH mechanism, represents the pushing of data or object to another module without the expectation of receiving any data from the method call. The standard form of this method call is "AcceptSomething", such as

"AcceptPurchaseOrder" or "AcceptProduct". We use a PUSH mechanism when sending data or an object to another module, such as warehouse delivering product to the customer. The prefix "Accept" suggests the method call does not expect a return value.

A third type of method call, an INTERNAL call, is private to the module. An INTERNAL call is initiated by a module to instruct itself to perform some action. The standard naming convention is to simply describe the action, such as the "GenerateDemand" method initiated by the Consumer module, or the "checkOrder" method within the Sales module.

A fourth type of method call, not demonstrated above and not yet implemented in the Simulation Builder, is the Delayed_PULL. In a Delayed_PULL, the accepting or called module would return a value after some elapsed simulation time. The calling module expects that some amount of time will pass, and suspends action until receipt of a response from the called module. Table 1 below summarizes the types of method calls. 
Table 1: Classification of Standardized Methods

\begin{tabular}{|l|l|l|l|}
\hline Method Type & Method Prefix & \multicolumn{1}{|c|}{ Action performed } & \multicolumn{1}{|c|}{$\begin{array}{c}\text { Simulation } \\
\text { time elapses }\end{array}$} \\
\hline PULL & Return & $\begin{array}{l}\text { Immediately returns a } \\
\text { value. }\end{array}$ & no \\
\hline PUSH & Accept & $\begin{array}{l}\text { Accepting module } \\
\text { performs an action but } \\
\text { does not return a value. }\end{array}$ & no \\
\hline $\begin{array}{l}\text { INTERNAL } \\
\text { PULL }\end{array}$ & (None) & $\begin{array}{l}\text { Initiated and performed } \\
\text { by same module. }\end{array}$ & possibly \\
\hline
\end{tabular}

\section{Structure of the Simulation Builder}

A module contains data structures and methods for executing its own internal operations, communicating with other modules, and for providing services to other modules. The particular data structures and methods needed to implement services particular to each module are not discussed here. We will discuss a set of data structures and methods modules require to communicate with other modules.

This section first discusses the way that the Simulation Builder organizes the services provided by the modules so as to allow the greatest flexibility to the designer in structuring modules. We follow this by an overview of the way we establish links between modules for each service. The last sections describe the $\mathrm{C}++$ classes used to implement the linking and communication.

\section{Organization of services}

The Simulation Builder's architecture allows the module designer the maximum flexibility in determining how modules should connect to obtain the services they need. It makes no assumption about how one particular type of module will obtain inputs from another particular type of module. For example, a module needs the service AcceptInstructionsShipProduct. Any assumption this service would always be supplied by a Warehouse module or by some derivative of a Warehouse module is untrue. Instead the designer might develop several types of modules capable of supplying this service. One might be a warehouse but another might be an order processing center. This gives the maximum flexibility in designing modules to build simulation models.

To accommodate this flexibility the Simulation Builder is partly organized around the services themselves rather than types of modules. Each type of service that can be supplied anywhere in the set of modules is defined as an entity in its own right-a separate class is defined for each type of service. If a module supplies a particular service, it inherits the capability to perform that service from the corresponding class. 


\section{Overview of the Structures and Methods for Communicating Between Modules}

A module calls methods in other modules to obtain services from them. To call a method in another module, a module needs a pointer to the other module. It can then make use of the pointer to obtain the service. When designing a module, the module designer will not know the names or the identities of the other modules with which a module will communicate. These modules will not be instantiated and identified until the instantiation of a particular model. Yet, a module's methods must make calls to these other modules. Our code provides a framework for a generic calls to, or a place holder for, a particular service provider. The needed pointers to other modules are made available at run time so that the actual calls can be made.

In designing the Simulation Builder, making the process of obtaining services as simple as possible for the module programmer is important. The mechanisms described below may be relatively complex to a programmer unversed in object-oriented programming. However, we implemented these mechanisms in classes which all modules inherit. They work in the background and the module designer does not to deal with them directly. The module designer essentially only specifies the particular type of service needed and implements a standardized class to make that service available to the module.

As was discussed above, the Simulation Builder is partly organized around services. One $\mathrm{C}++$ class exists for each type of service defined. We refer to one of these classes as the "standard service message class" (SSMC) for the service. For example, there is a standard service message class for ReturnInventory. It will typically be written as ssmc_ReturnInventory. A module class inherits an SSMC for each service it wants to provide. Thus, when a module class inherits the SSMC for, say, ReturnInventory, it inherits a virtual method for the service. It then redefines (overrides) the method for its own situation.

This approach has the advantage in that it allows the system to compile a registry of all of the modules that have inherited a particular SSMC and, therefore, can provide the corresponding service. This registry of modules is key to establishing communication and to checking the validity of the links established. A later section discusses the method in which the registry establishes itself.

The actual connection between modules is handled by "Ports" and Providers". These are very closely related. A module implements a link to a particular service by including as an attribute a Provider of the correct type. Thus, when the code in one of the module's methods needs a particular service (supplied by another module instance), the code simply calls the corresponding Provider, asking for the service. The Provider has the methods and data needed to obtain the service. A Port is part of a Provider (technically, a Providers inherit from a Port). The Port contains the methods needed to find a pointer to the external module supplying the service when the model is instantiated. The Port also checks the validity of the connection at the time of instantiation.

A different Provider class exists for each type of service in the model. Thus, a one-to-one correspondence between the Provider classes and the standard service message classes exists. Each Port/Provider has access to the registry of modules that provide its particular service.

When a module is first constructed, it reads the input name of each module that will provide a service to it (these would be listed in a file by the user). The module constructs its Providers. Each Provider is initialized with the input name of the module from which it will obtain the service.

Figure 2 and Figure 3 provide a concrete illustration of the way that the modules are structured and the mechanism for linking them. Here the module named OurSales 
requires the service ReturnInventory. The module instance named CentralWarehouse will supply this service.

Figure 2 shows the state of the modules immediately after they have been constructed. The registry of modules providing the service ReturnInventory includes an entry for CentralWarehouse. This entry provides a pointer to CentralWarehouse. Within OurSales, a Provider for the ReturnInventory service was constructed when OurSales was constructed. One of the parameters of the constructor for OurSales was the input name of the module to provide the service. This name is passed to the Port during initialization. Since the Provider is the type that corresponds to the service ReturnInventory, it has a reference to the registry of all modules in the model that can supply that service. At this point, however, the pointer attribute of Port/Provider has not been initialized to the module that will supply the service.

Figure 2: State of modules and registry after the modules have been constructed but before connecting

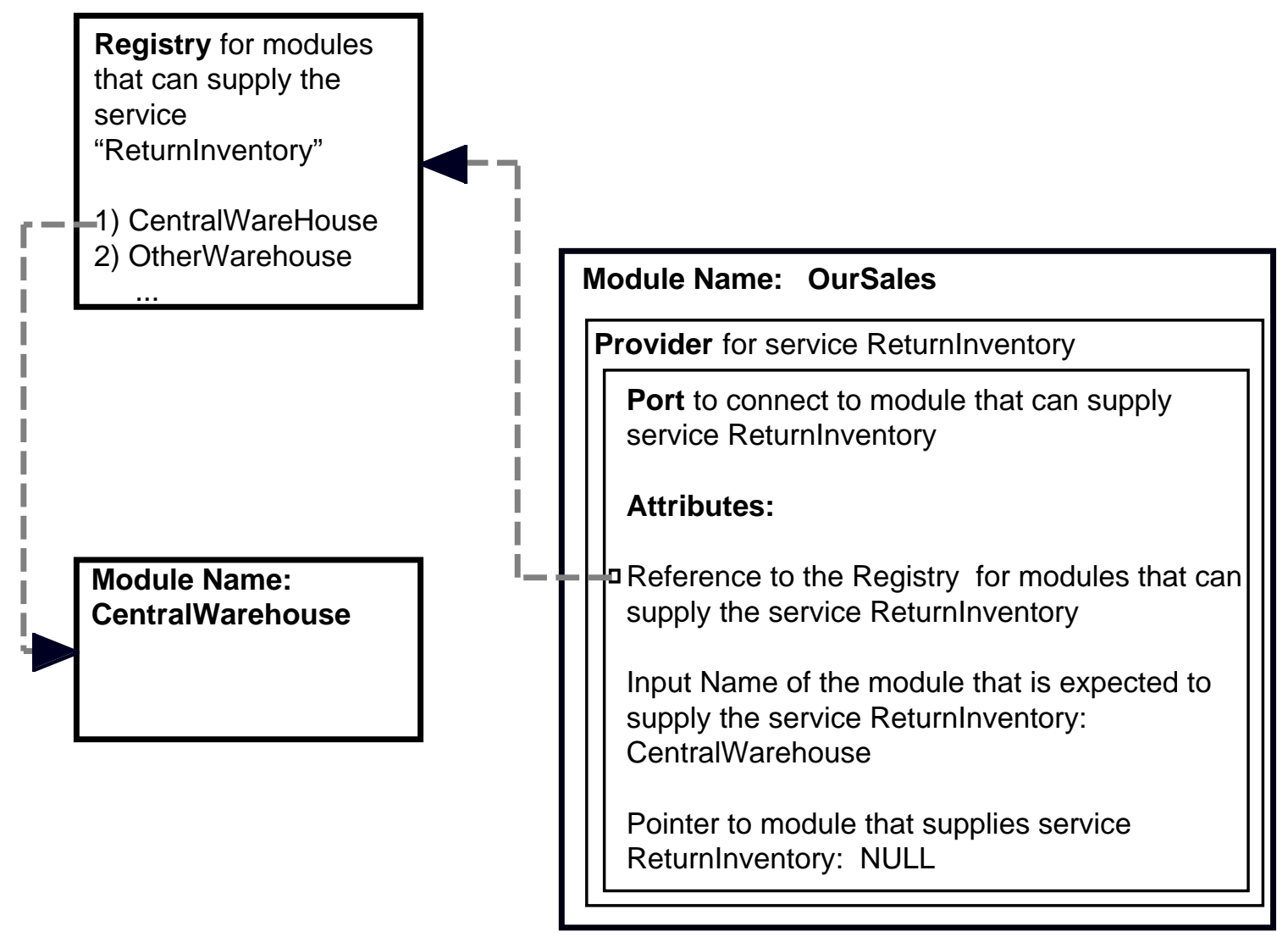

To obtain the pointer and establish the link we invoke the connect() method in the Port. The connect method finds the module by checking the registry for a module with the input name "CentralWarehouse". Once it finds the module, it retrieves a pointer to the it. At this point the Port/Provider pointer is initialized as is shown in Figure 3 
Figure 3: State of modules and registry after connecting

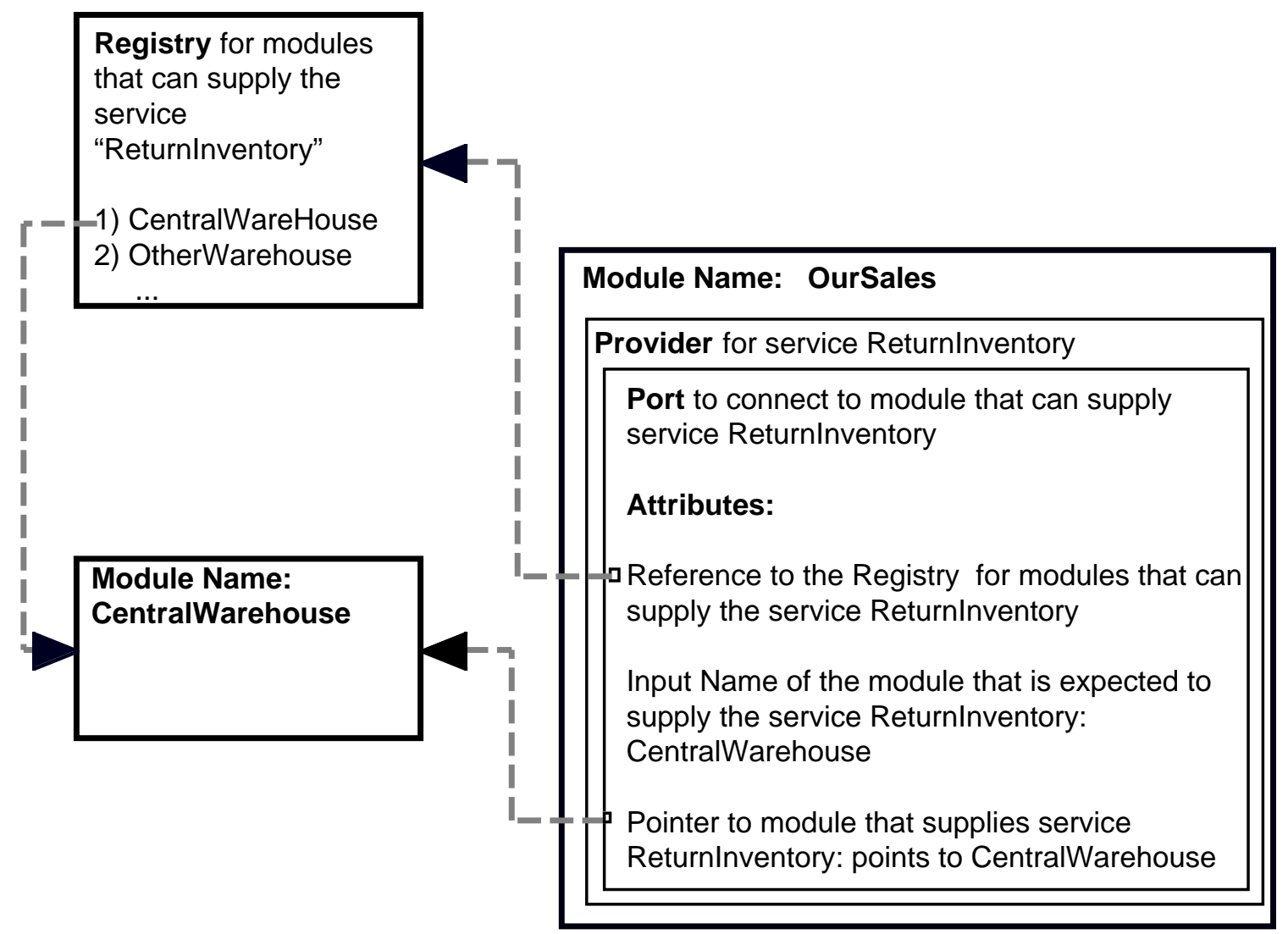

This has provided an overview of the mechanism for linking modules. The sections below provide more detail about each of the classes involved.

\section{Standard Service Message Classes (SSMC)}

We implement the services that a module provides through the mechanism of a standard service message class. One defines an SSMC for each type of service (implemented as a method) a module may provide. A given module provides only a subset of possible services.

A module class provides service by inheriting the SSMC corresponding to that service. The SSMC establishes only a "virtual" version of its method. A module must override the method with its own implementation. The specific implementation of the service (method) will then be defined at the module level. This allows the Simulation Builder to supply a number of different modules that provide a certain service, such as the service ReturnInventory(). Thus, each module may implement the method differently, according to the data structure available within the module type. This allows the hiding of implementation detail.

In general, we group class attributes (attributes shared by all instances) into a single class, which we name the class attribute class (CAC). We denote class attribute classes by prefixing the class name with "CAC_". For instance, CAC_ssm is the name of the class attribute class within any SSMC. The CAC is a static object that "is a" (in a relational 
sense) list of all module instances which can provide the service corresponding to the SSMC. At instantiation, each SSMC instance registers itself on this list.

The objects pointed to by the CAC_ssm list are of type instance_info (short for instance information). The instance_info_mod class holds the instance information for a module. All modules must inherit from the instance_info_mod class. The instance_info_mod contains a place holder for the input information, including the user specified input name. It also contains a pointer to the module.

For convenience and simplification of programming steps, we aggregate all the services we want a particular module type to supply into a single conglomerate SSM class. The module designer must create this aggregate SSM class. Figure 4 below shows the services that a module_warehouse class supplies conglomerated into the SSM class "ssmc_warehouse". The "ssmc_warehouse" class inherits from the classes "ssmc_ReturnInventory", "ssmc_AcceptProduct", and "ssmc_ShipProduct".

In Figure 4, the full "module_warehouse" class inherits from the conglomerate ssmc_warehouse class and the generic module class.

Figure 4: Example inheritance structure for module type module_warehouse.
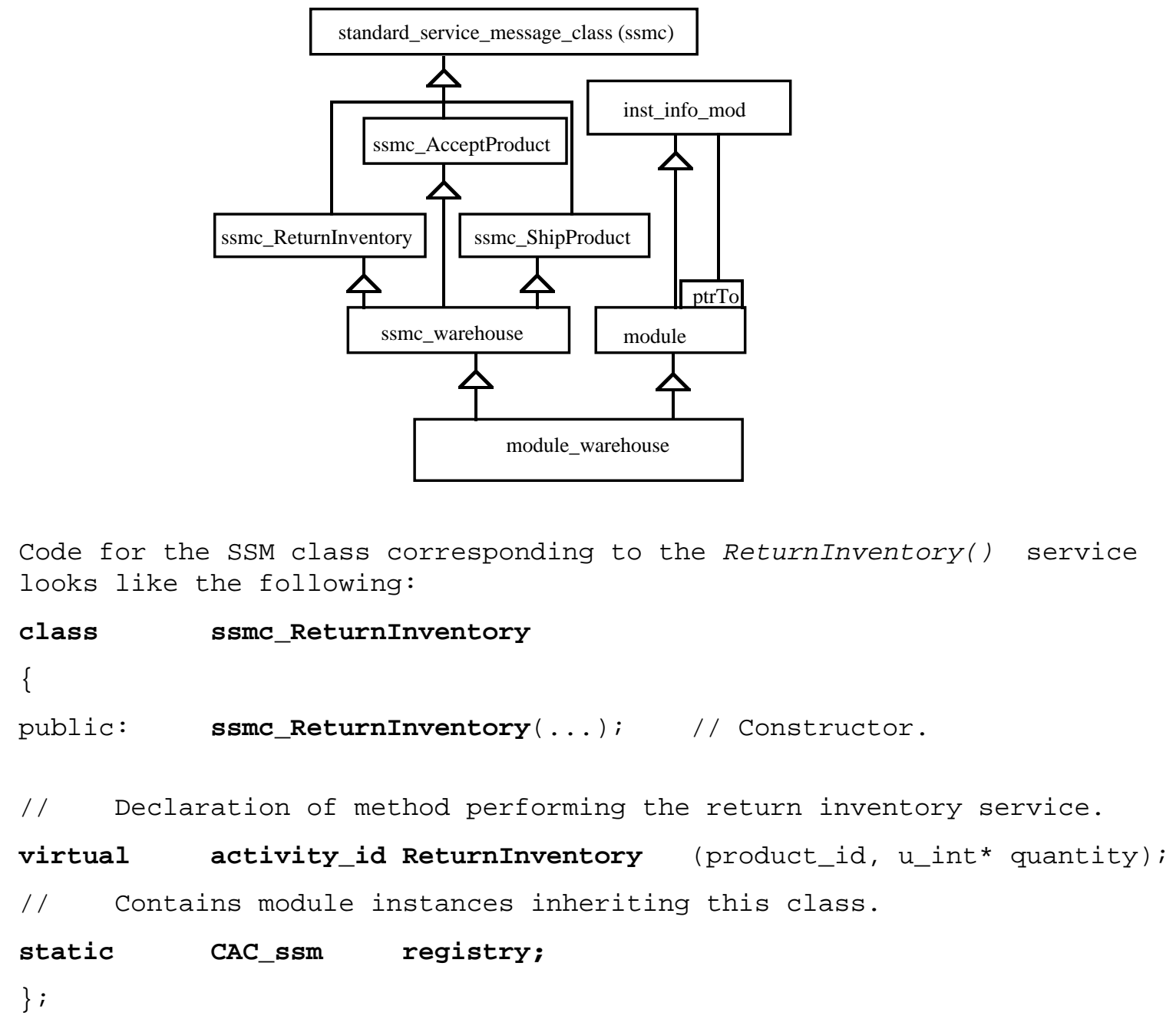

One constructs a conglomerate standard service message class for a module type from the set of SSMC's corresponding to the services a module type provides. For example, for a 
module that ships product, returns inventory, and accepts product we would construct the following:

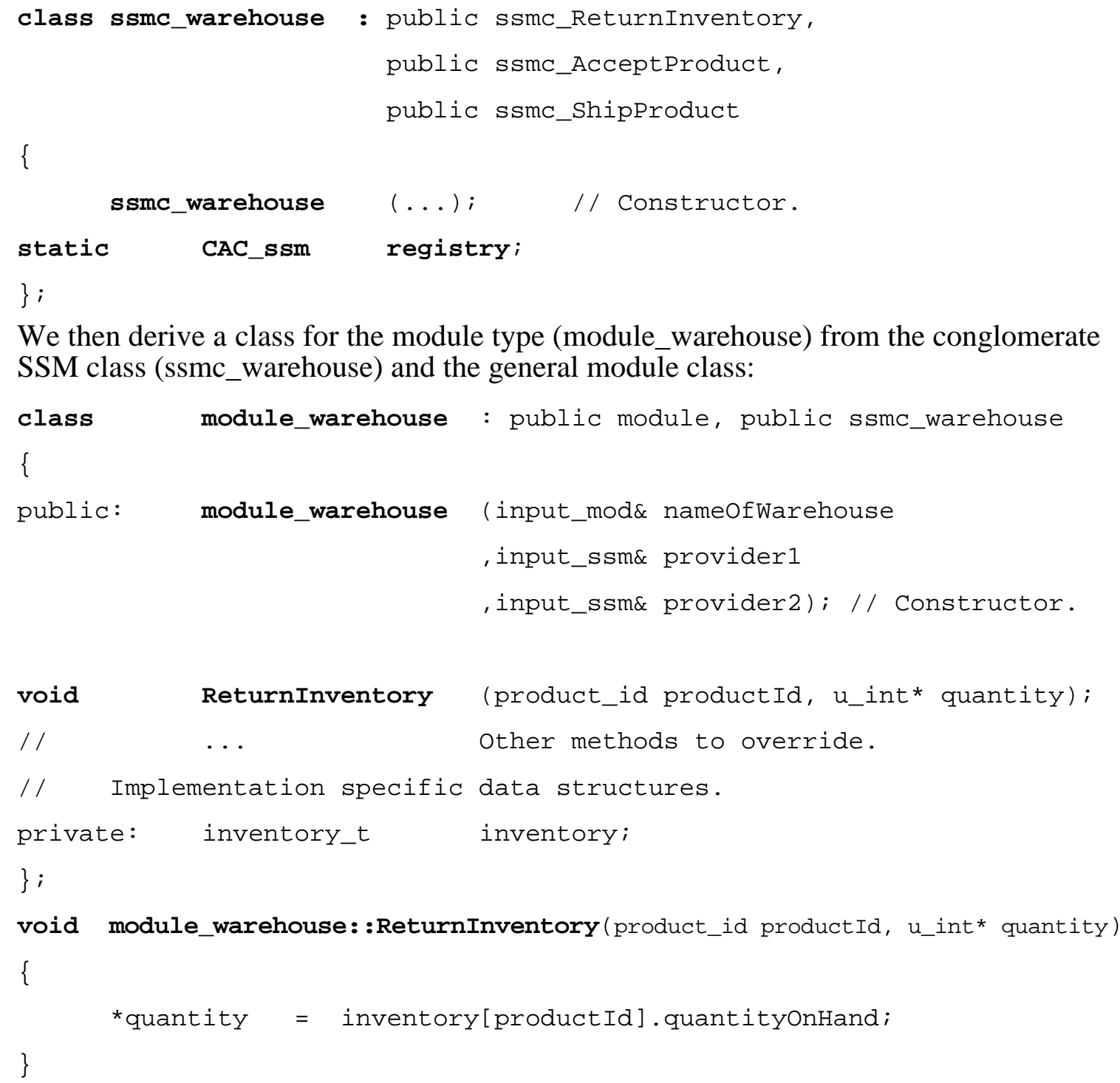

We then derive a class for the module type (module_warehouse) from the conglomerate SSM class (ssmc_warehouse) and the general module class:

Section IV provides a set of MACROs specifying the code for these classes in their entirety.

\section{Ports}

The "Port" object establishes the connection between a module that supplies a service and a module that requires a service. The Port contains the type independent attributes used to support links. The Port has a reference to the object containing the input name of the module that will supply a service. It also has a reference to the CAC_ssm for the class of objects that can supply the service. The Port includes a method for obtaining a pointer to the module bearing the input name.

When designing a module, we need to include a Port/Provider attribute for every service the module (being designed) needs from external modules. The Port abstraction provides one level of indirection to the service-supplying module. Initially, the Port holds only the "input name" corresponding to the object/module supplying the service. Due to the unpredictable order of module instantiation, the simulation may have yet to instantiate the 
module instance of interest. A Port mechanism allows the module that owns the Port to later connect itself with the service-supplying module.

The Port contains the type-independent data which the Provider class will inherit. When instantiating a module, each Provider attribute in turn instantiates a Port. The Port constructor gets passed three references as arguments, one to an input_ssm, a second to a CAC_ssm and a third to an instance_info_mod. The input_ssm contains, as an attribute, the input name of the module to supply the service. The CAC_ssm is the class attribute data listing all of the modules that can provide the service. The instance_info_mod contains a reference to the module that owns the Port.

At instantiation, the Port constructor registers the Port with the list of all ports. This registry, called CAC_port in the code implementation, is a static data structure shared by all instances of Port. The CAC_port contains the additional method Connect(). When this static data structure receives the connect message, it in turn sends the message to all ports in its registry of ports.

When a Port receives the connect() message, it executes the following steps, as illustrated by Figure 5:

1) Retrieves the input name of the module it will connect to from the attribute of type input_ssm, named "input".

2) Sends a find() message to the CAC_ssm that corresponds to its type of service. The find() operation takes as an argument the input name of the module to connect. At instantiation, this CAC_ssm class was supplied to the Port by the Provider inheriting this Port. (See section III-D-4, Providers). The CAC_ssm class has a find () method. The CAC_ssm class searches its list of instance_info_mod. If a match is found within the list, find () returns a pointer for the module instance. If no match, find () indicates an error. A non-match indicates either that the module "Name" never registered itself with the service class (and thus, cannot supply the service), or that the "Name" was misspelled.

3) Takes the pointer find() returns and it pointer in the "link_ptrTo" attribute of the Port.

Figure 5: Connection between modules

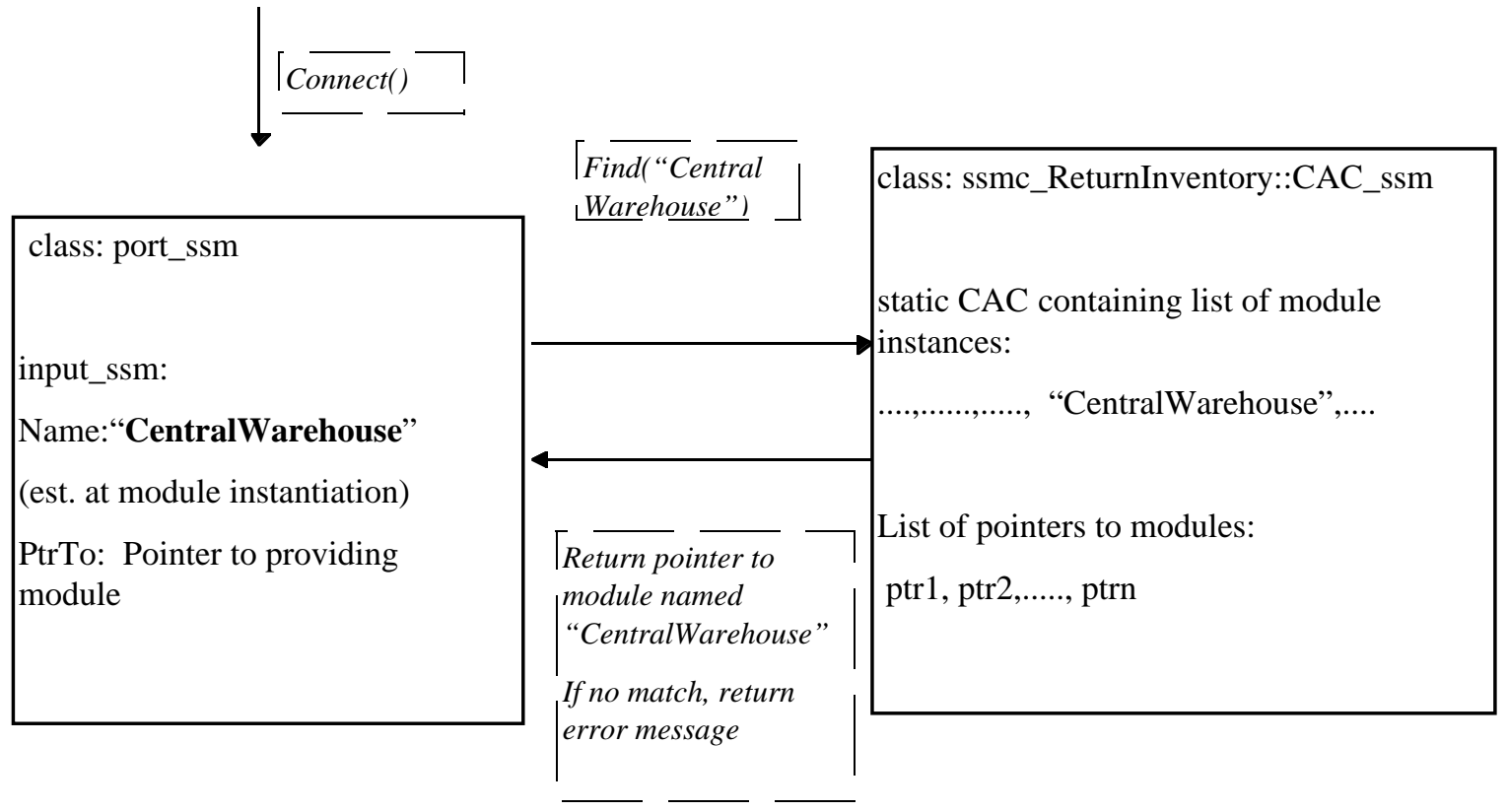


Note the Provider supplies the Port with the proper SSMC class attribute class (CAC). The following code defines the Port class:

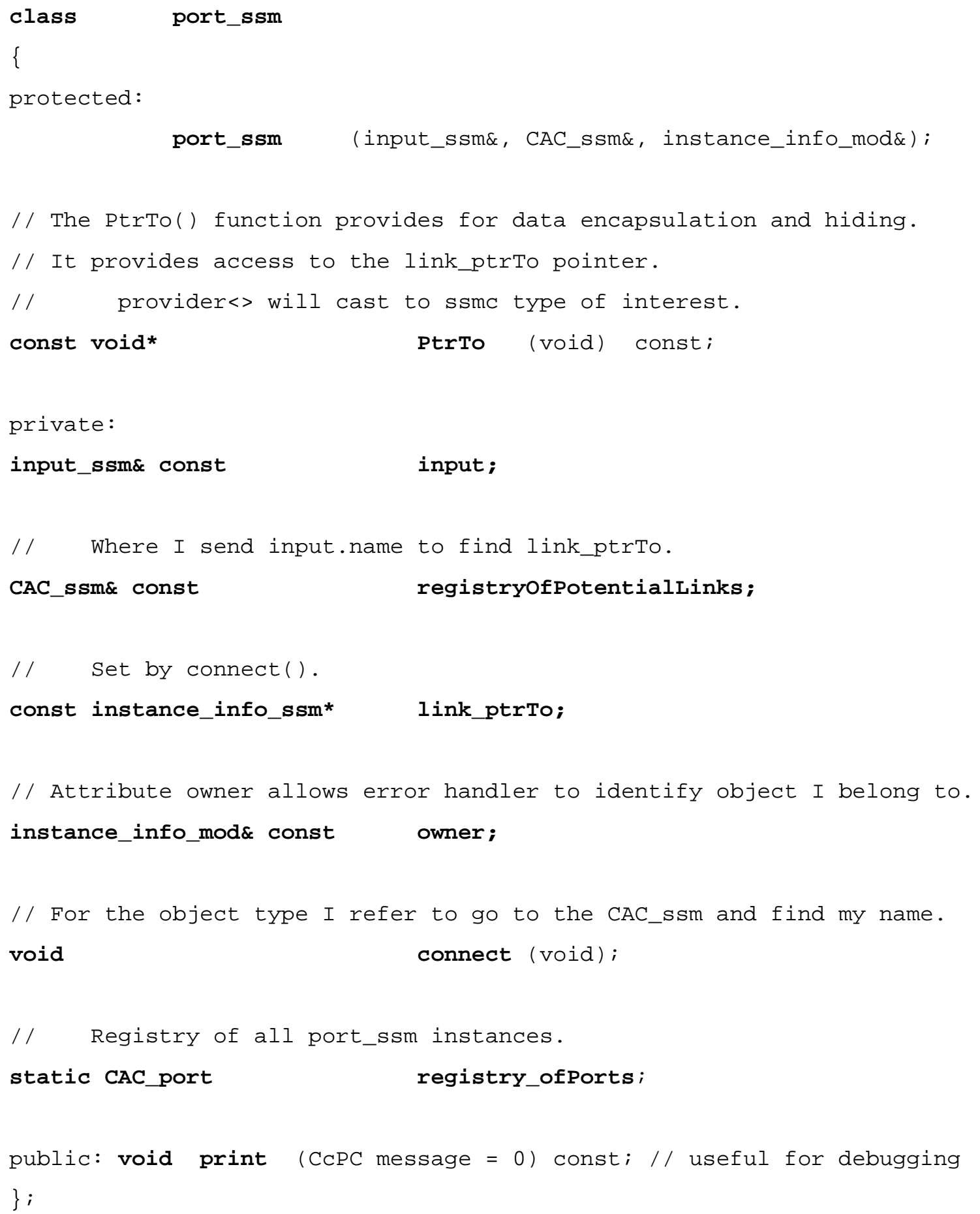

Figure 6 shows the inheritance relationship between standard service message classes (ssmc's), modules, and Port and Providers. 
Figure 6: Inheritance diagram showing the relationship between module, standard service message class, and ports

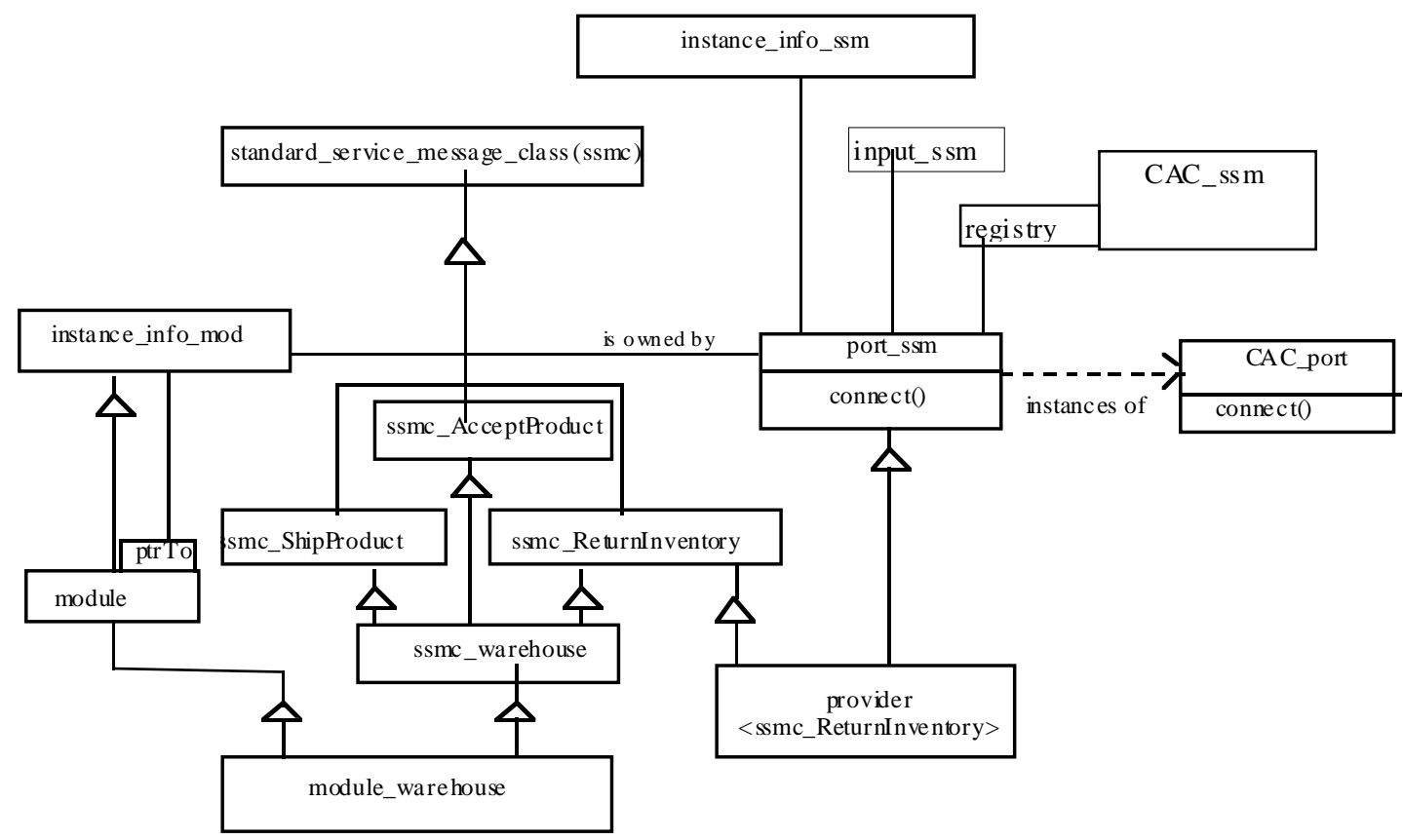

\section{Providers}

A "Provider" inherits a Port, and is the mechanism with which a module can access a service supplied by another module. Within a module class we include as attributes a set of providers which will provide the module with the services it requires. A module that requires a service need only ask the corresponding Provider for the service. For example, a module that requires a report of inventory level from its associated warehouse, need only ask its attribute of type provider<ssmc_ReturnInventory> to ReturnInventory. The specific name of the supplying module (such as "SupplierWarehouse" or

"WestCoastWarehouse"), need not be known to the module making the request. The Provider, therefore, acts as the place-holder.

The Provider, at instantiation, is passed the name of the service-providing module, and, therefore, can ask the providing module for the service. This implements a level of indirection such that the specific instance that supplies the desired functionality (the service) need not be named directly by the calling module.

A Provider is an attribute found in a module. A module must include a separate Provider attribute for each service it requires. As an extension of a Port, a Provider inherits from both the Port object and the SSMC corresponding to the service required. For ReturnInventory(), for example, we define the Provider class which inherits from the generic Port class (port_ssm) and the SSM class corresponding to ReturnInventory. 


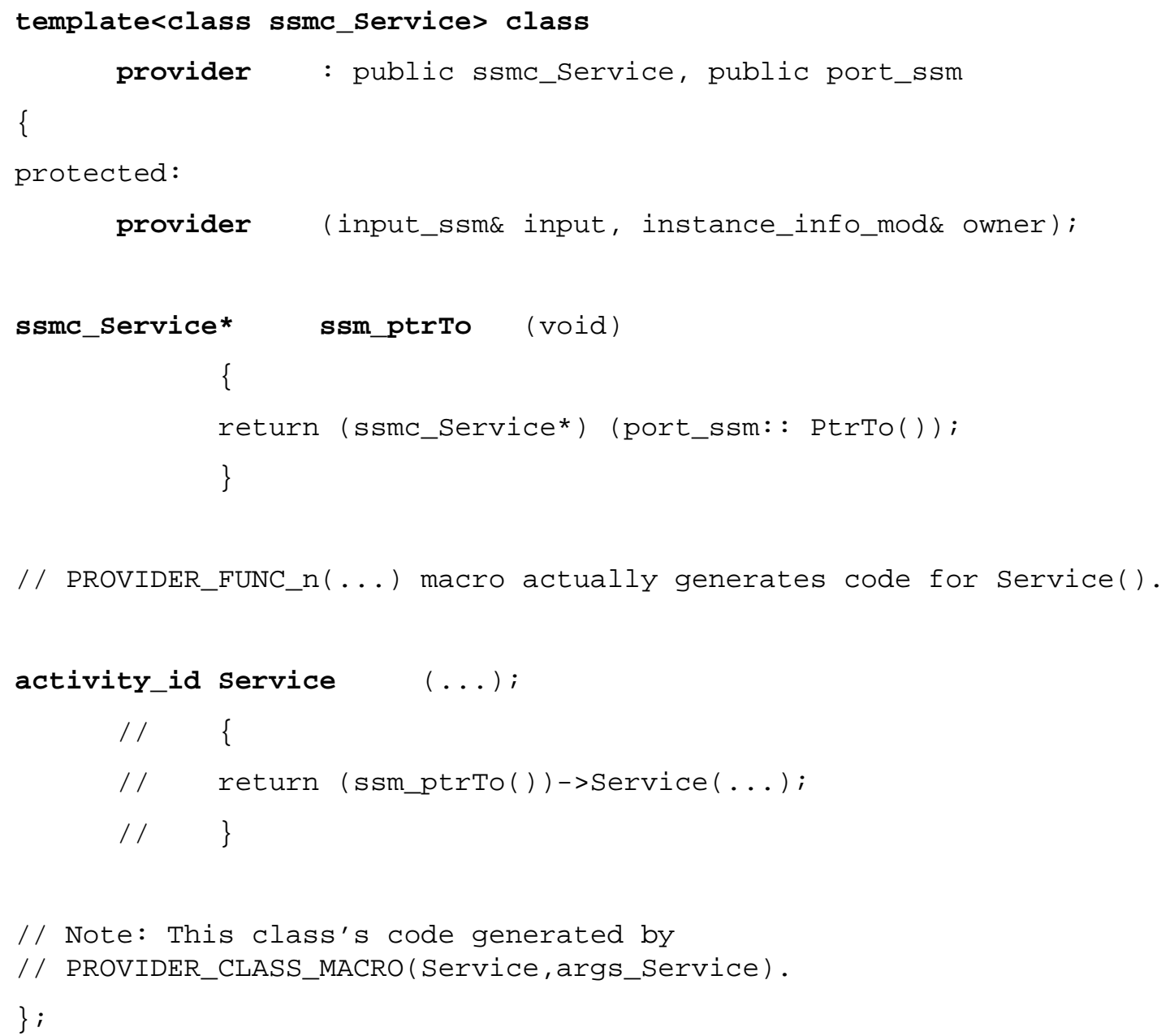

A constructor for this Provider instantiates the Provider, passing it a reference to the object containing the name of the module to link to, as well as a reference to the module containing said Provider. A macro exists to specify all but one code statement needed to implement a Provider class.

The Provider class inherits a standard service message class and a Port. A Provider adds the notion of type, with respect to the standard service message it provides. A Provider that inherits an SSMC, say ssmc_Service, is said to be of type ssmc_Service. A Provider then casts the generic pointer obtained from the Port (from which the Provider inherited) to its SSMC type. It supplies its Port with the proper SSMC class attribute class. The Port contains no concept of type other than the class attribute class it is given. The Provider redirects a standard service message sent to it from the module which owns it, to the module the Provider obtained through the connect() method.

A module that requires an inventory Provider would then initialize an object of type, provider<ssmc_ReturnInventory>: 


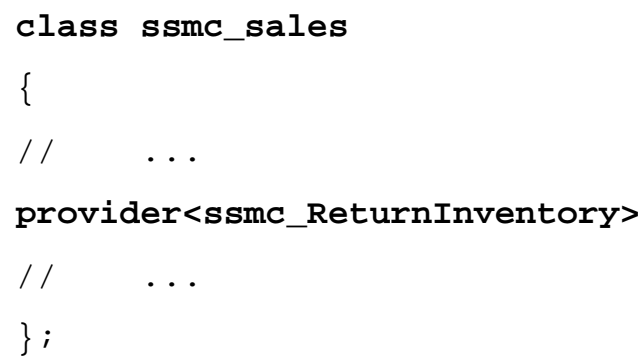

ReturnInventory_provider;

and then access the service in the following way,

ReturnInventory_provider.ReturnInventory (productId, quantity_ptrTo);

Before the Provider can actually access the corresponding function in the providing module it must cast it to the corresponding SSMC. The Port is supplied with the proper SSMC class attribute class (CAC) by its provider. The Provider casts the "PtrTo()" pointer to its SSMC type. Figure 7 illustrates this step.

\section{Figure 7: Pointer returned from find() message is cast to the appropriate SSMC class}

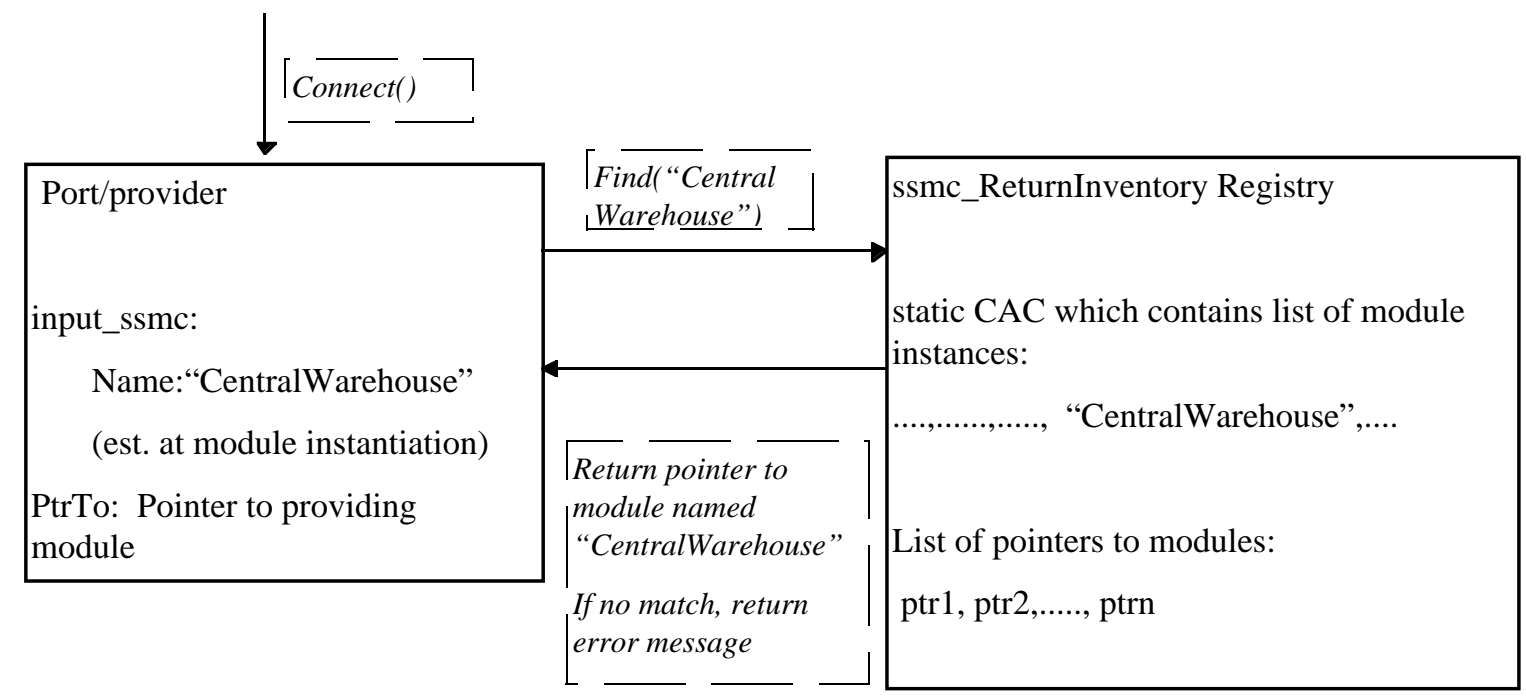

\section{E. Validity Checking through Module Registry}

To insure the feasibility of a set of modules defined for a simulation model, validity checking on model structure must be performed by the Simulation Builder. We establish a mechanism to maintain a registry of module instances. The registry keeps track of the existing module instances for a simulation model, and can be used to check for valid module connections. A link between modules is valid if it connects a module requiring a service to a module which can provide that service. Note, several modules in a model may provide a particular service. The simulation cannot check that the connection is to the correct module, just that the module specified can supply the service. That is, if one specifies the wrong name, but the name is the name of another service supplied of the same type, an error would not be indicated. 
The modules supplying a service type are maintained in a registry for that service. One registry is established and maintained for each type of service. Recall, this registry is the CAC static data structure for the SSMC. The registry is a list of modules that inherited the standard service message class, and therefore, can supply the service. The service registry contains the user-specified names of the module instances and the pointers to the modules. At instantiation, the constructor of a module class is called, and the module registers itself with each SSM class it inherits. We instantiate an object holding the instance information for a module (defined as instance_info_mod, which contains the name and pointer to the module) as part of a module. The module being constructed passes its instance_info_mod object to the constructor of each SSM class that the module inherits. Through this mechanism, the SSM classes generate the lists. From the list, the simulation can identify the instances implementing the corresponding service.

\section{F. Model Instantiation}

One can instantiate a model from an input file containing a list of module descriptors. At some later time it is expected that the Simulation Builder will be added to a system that will include a graphical user interface for constructing models. Under the current approach, a module input file may consist of module entries as follows:

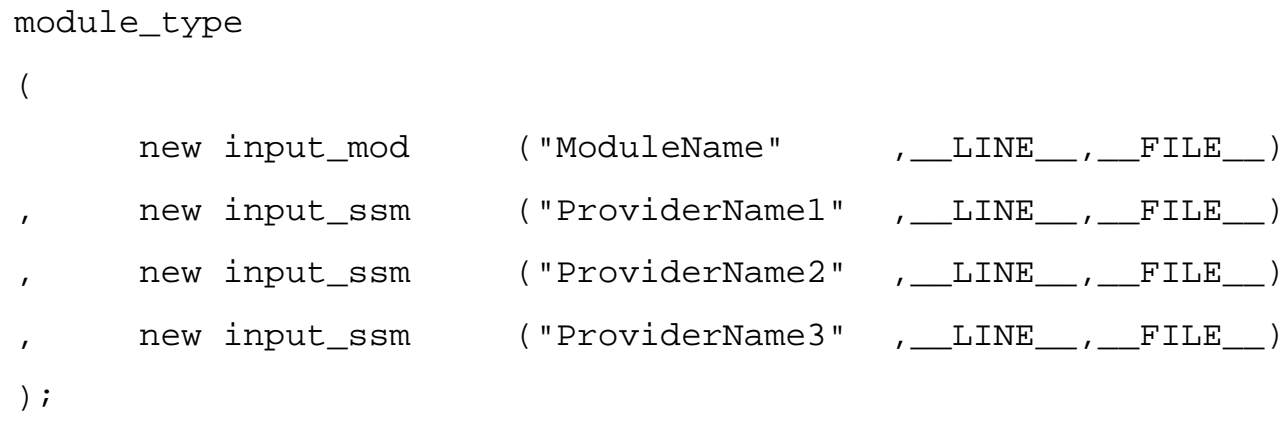

The constructor of a module class expects an input_ssm for each service it will supply. A constructor for module warehouse would take as arguments, input_ssm's with the literal string name of the warehouse, and literal name of provider1 and provider2. Module_type refers to the type of the module (e.g., Warehouse, Sales, etc.), which must be defined. The first argument is input_mod associated with the name of the particular instance. The remaining arguments indicate Providers. Note, the entry must list the Provider names in the order defined by the module_type constructor. The $\mathrm{C}$ preprocessor MACROs _ LINE_ and _ FILE_ _ pass information pertinent to locating the error in the input file.

After the instantiation of all modules, the system establishes the connections between modules and the "Provider" class instance. Recall that each Provider type class inherits from the class port_ssm. Also recall the CAC_port maintains a list of all ports. When the constructor for the Port is called, it adds the Port (for the Provider) onto the list of all ports maintained in the CAC_port class.

When the CAC_port class receives the Connect() message, it sends a Connect() message to each Port in its list. When a port_ssm receives the connect message, it passes its literal name to the CAC_ssm and asks the CAC_ssm to find name in its list. The find () function searches through the list of instances of the modules, searching for a name that matches. If a match is found, a pointer to the module instance is returned. If no match, the program indicates an appropriate error message. 


\section{Steps for the module designer}

When writing code for a new module, the module designer determines the functionality that the module will provide the simulation. The designer will specify the module as a set of services, using pre-defined services, if possible. For an undefined service, s/he will create a new service.

The generalized Simulation Builder library provides a set of MACROs to facilitate the design of new modules and new services. Section IV-A describes how one would use these MACROs to create a new service for use in an existing module, or for the creation of an entirely new module. Section IV-B describes the steps to follow for the creation of a new module from the library of existing services.

\section{A. Creating and Implementing a New Service}

In designing a new service, the designer will

1) Develop the standard service message:

Determine the function prototype for the service. Specify its name and optionally any input and/or output arguments. If no inputs or outputs, specify void for the argument list. All services will return the type activity_id. A prototype function would look like the following:

activity_id AcceptPurchaseorder (inputs_t, outputs_t*);

Using this function prototype create the SSM_Service macro for the service. Note, we prefix the service with SSM (for standard service message) so that we can readily identify the method as one of the standards.

\#define SSM_Service Service (inputs_t, outputs_t*)

Example:

\#define SSM_AcceptPurchaseOrder AcceptPurchaseorder(inputs_t,outputs_t*)

2) Define the corresponding primitive SSMC:

Every service requires the definition of a corresponding primitive SSMC (PSSMC). Primitive class as distinguished from conglomerate SSM class -- a class inheriting more than one primitive class. For example, the conglomerate class ssmc_warehouse inherits the ssmc_ReturnInventory, ssmc_ShipProduct, and ssmc_AcceptProduct classes.

a) Use the macro PSSMC_MACRO $(x, y)$ to define the PSSMC. The first argument specifies the name for the PSSMC, the second must be the SSM macro for the service defined in step (1) above.

PSSMC_MACRO (Service, SSM_Service)

Example:

PSSMC_MACRO (AcceptPurchaseorder, SSM_AcceptPurchaseorder); 
The PSSMC_MACRO(Service, Service_w_arglist) MACRO definition is:

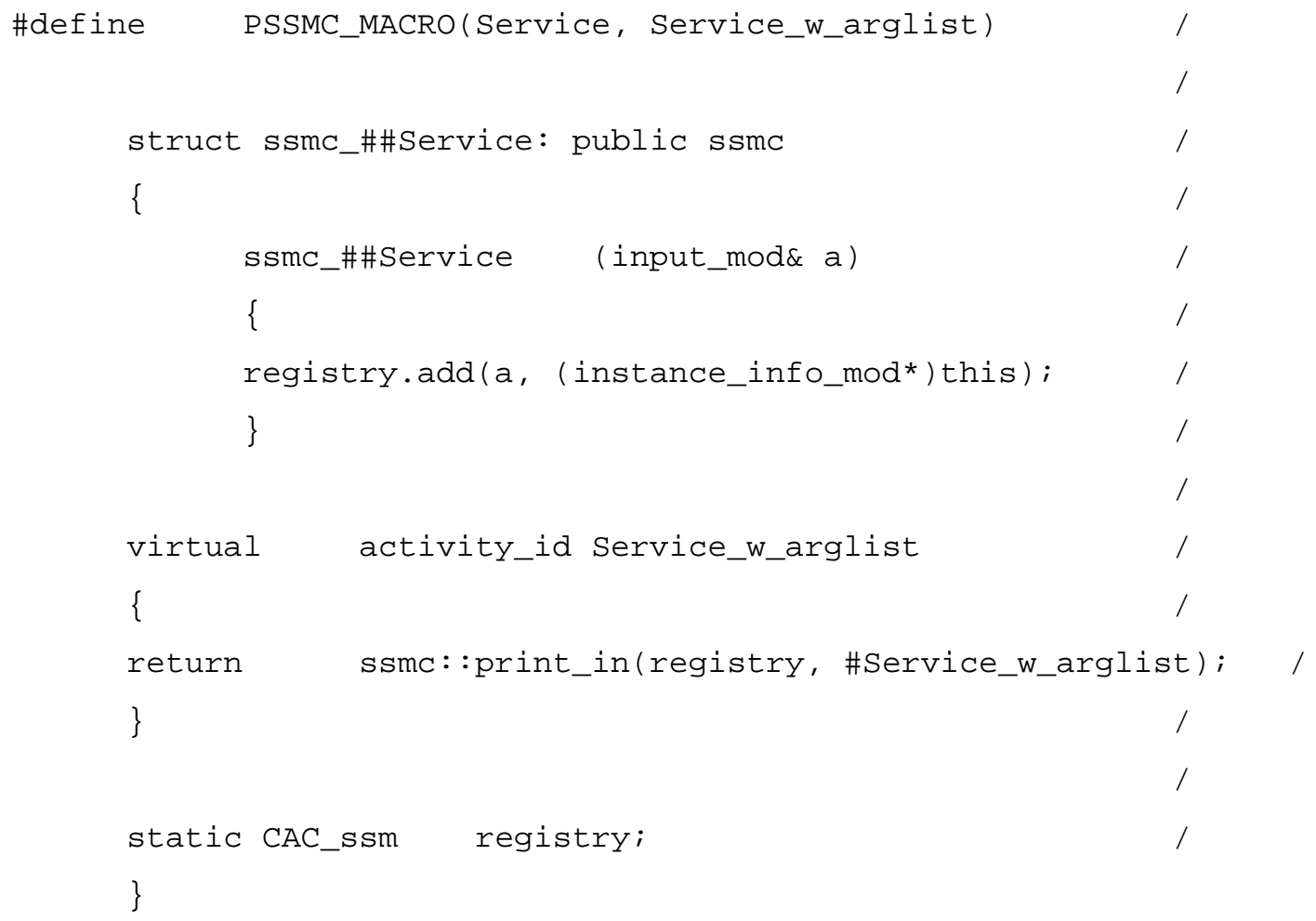

b) For every PSSMC_MACRO entry, add a CAC_SSMC_MACRO(), using Service as a parameter. This step takes the literal string defined by Service, and passes the entire string as a parameter to the CAC static data structure.

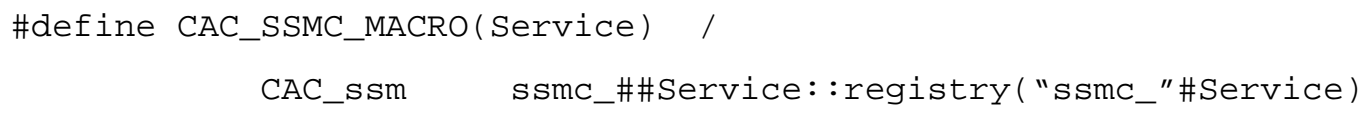

For example:

CAC_SSMC_MACRO (ReturnInventory);

After preprocessing CAC_SSMC_MACRO (ReturnInventory); expands to

CAC_ssm ssmc_ReturnInventory: :registry ("ssmc_ReturnInventory");

3) Develop the corresponding Provider class for the service.

The Provider class will inherit from the SSMC class.

a) Given ssmc_Service defined above and the name of the Provider, use the PROVIDER_CLASS_MACRO.

PROVIDER_CLASS_MACRO (Service, SSM_Service) 
For example:

PROVIDER_CLASS_MACRO (AcceptPurchaseorder,SSM_AcceptPurchaseorder)；

b) Unlike the PSSMC_MACRO which specifies the code in its entirety, the Provider macro leaves a step for the designer in that it doesn't specify all the code. The designer must type in a single stub of code that looks like the following:

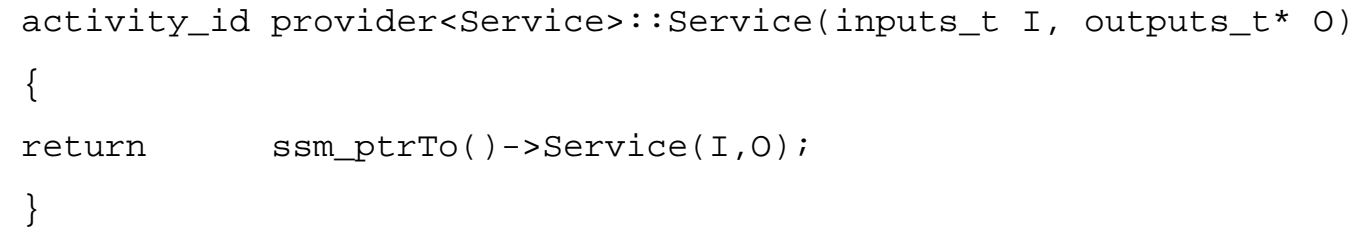

Note that the input and output argument list must correspond to the input and output list in Section IV-A-1.

\section{B. Designing a New Module}

1) Implement services for the module.

a) Identify the services that the module will provide. If service exists, use existing service.

b) If service does not exist, define new service as described in section IV-A.

c) Establish conglomerate SSM class for the module (ssmc_module_type). The class ssmc_module_type will inherit from each SSM class that corresponds to a service.

d) Create a module from the set of pre-defined services.

i) Use one of the SSMCM_MACROn's to specify the conglomerate services (SSM classes) a module will provide. The SSMCM_MACRO establishes the ssmc_module as a "provider" of the particular service.

The code SSMCM_MACRO3 (ssmc_conglom,ssmc0,ssmc1,ssmc2); expands to:

class ssmc_conglom : public ssm0, public ssm1, public ssm2

$\{$ protected:

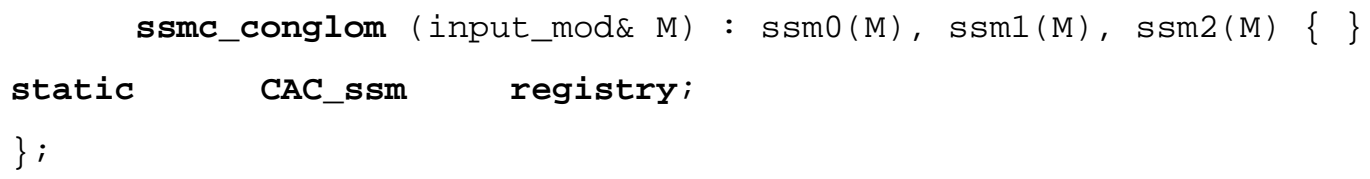


ii) If the module uses services, create a conglomerate macro for the services a module will use:

\#define SSM_module_name SSM_service1,SSM_service2

For example:

\#define SSM_Sales SSM_AcceptPurchaseorder, SSM_ReturnSalesHistory

\#define SSM_Warehouse SSM_AcceptReceiveProduct

e) Define a module class that inherits from the module super class and the ssmc_module_type class.

For example:

class module_warehouse : public module, public ssmc_warehouse

f) Define the constructor for the module.

g) Override the methods for each service.

2) Define the modules that will use the services provided by this module.

Each module that uses the service must have a Provider defined within it so that it can access the service.

a) Define a Provider class that inherits from the ssmc class. (This step was already discussed in section III-D-3.)

PROVIDER_CLASS_MACRO (ReturnInventory,SSM_ReturnInventory);

PROVIDER_FUNC_1 (ReturnInventory, product_id, u_int*) 
Example:

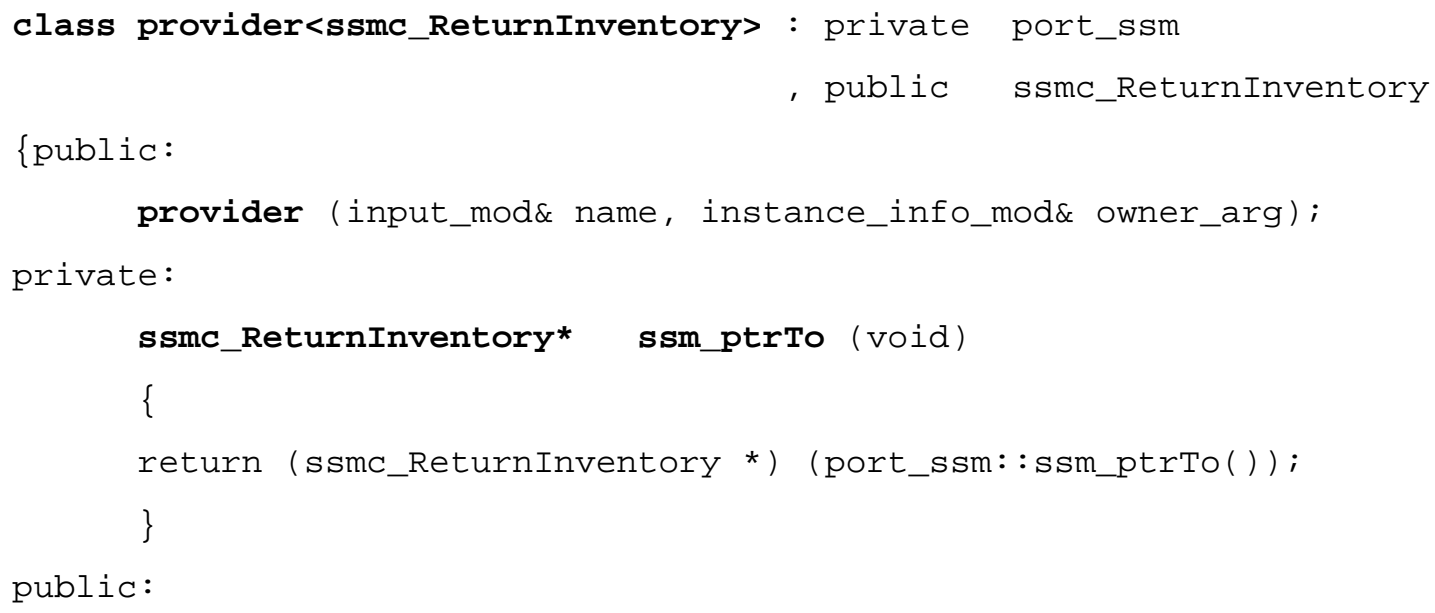

b) Include the Provider in the module class description

For example:

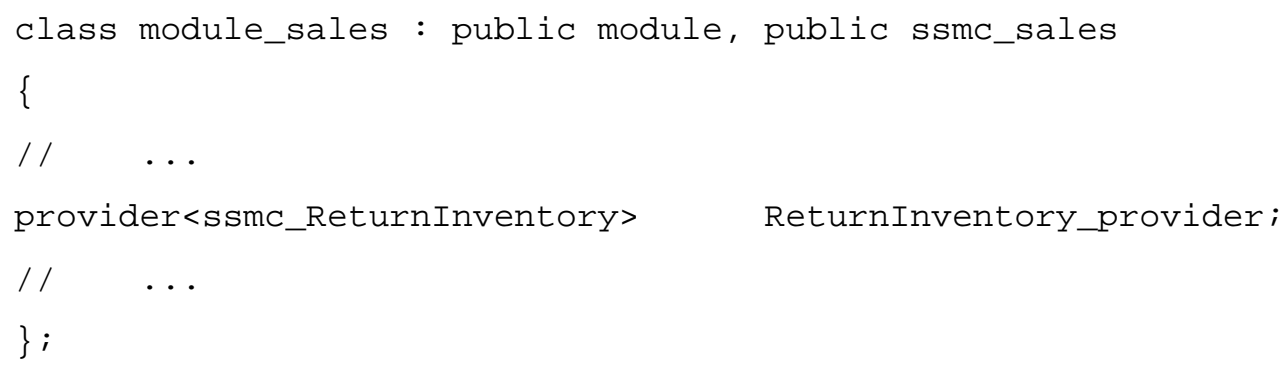




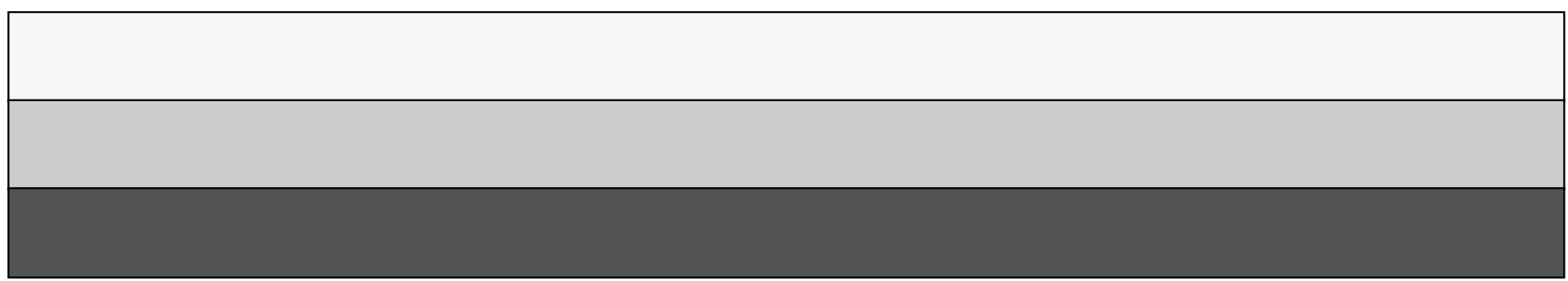

